\title{
A view of image denoising method based on Metropolis light transport
}

\author{
Huaiqing $\mathrm{He}^{*}$, Jiaqian Yang, Haohan Liu \\ Department of Computer Science and Technology, Civil Aviation University of China, Tianjin, China \\ Email:631906692@qq.com
}

Keywords: Metropolis; ray tracing; sampling; image denoising; Monte Carlo.

Abstract. Metropolis light transport was an unbiased and robust Monte Carlo method, which could efficiently reduce noise during rendering the realistic graphics to resolve the global illumination problem. First, described basic Metropolis method, and then focused on its own characteristics to outline typical research progress based on the main algorithm influence factors in recently years. Finally, proposed the next plan after summarizing its applications effect in the scenes.

\section{Introduction}

The main objective of computer graphics is to do realistic graphics rendering with different models in complex illumination. Monte Carlo as proposed by Kaijia et al. [1] to solve reflections, indirect illumination and complex scenes in global illumination with simplicity and adaptation. Monte Carlo light transport using light to discretely sample for solution space of rendering equation. Path Tracing (PT), Bidirectional Path Tracing (BDPT) and Metropolis light transport (MLT) [2] are derived from Monte Carlo light transport. PT and BDPT are simple sampling methods, and randomly sample paths from light resource to eye without consideration of path distribution. Uneven path distribution will reduce the efficiency greatly, and does not make full use of the similarity between samples [3]. Metropolis method initially addressed complex sampling in physical field proposed by Metropolis et al at. [4], then Veach et al. use it to do adjustments for known paths with similarity. The paper focus on the basic MLT and typical improvement based on the main factors in the recent years.

\section{Basic Metropolis light transport method}

\section{Basic Metropolis sampling method}

Basic form of Metropolis sampling

Supposing a nonnegative function $f: \Omega \rightarrow \mathbb{K}^{+}$defined in sample space $\Omega$ and generating a sequence of sample $\mathrm{X}_{0} \ldots \mathrm{X}_{\mathrm{i}} \ldots$ with probability density function $p_{i}$, given the initial sample $\mathrm{X}_{0} \in \Omega$. Each sample $\mathrm{X}_{\mathrm{i}}$ is obtained by choosing some mutation strategies, as long as it satisfies ergodic. The mutation strategy is described by tentative transition function $T(\mathrm{Y} \mid \mathrm{X})$ [5]. Desired sample distribution is obtained by accepting or rejecting proposed mutations according to a chosen acceptance probability. Defining $a(\mathrm{Y} \mid \mathrm{X})$ as the acceptance probability for accepting mutation $\mathrm{Y}$ as $\mathrm{X}_{\mathrm{i}+1}$, given $\mathrm{X}=\mathrm{X}_{\mathrm{i}} \cdot a(\mathrm{Y} \mid \mathrm{X})$ satisfy the detailed balance condition[6]:

$$
f(X) T(Y \mid X) a(Y \mid X)=f(Y) T(X \mid Y) a(X \mid Y)
$$

The best strategy is to make the acceptance probability as large as possible. Then results in:

$$
a(Y \mid X)=\min \left\{1, \frac{f(Y) T(X \mid Y)}{f(X) T(Y \mid X)}\right\}
$$

Expected values

To get the change information about $f(\mathrm{X})$ 's behavior in such regions, the expected values technique can be used to enhance the basic Metropolis algorithm. The current sample $X$ and proposed sample $\mathrm{Y}$ will be record at each mutation with a weight associated with it. Where the weights are the probabilities (1-a) for $\mathrm{Y}$ and $a$ for $\mathrm{Y}$, and $a$ is the acceptance probability.

\section{Basic Progress of Metropolis light transport}

Defining $\Phi\left(S_{i}\right)=\int_{\Omega} f_{i}(X) d \mu(X)$ as the radiant flux of path from light resource to pixel $j$ according to 
the path integral form of rendering equation [1]. Then divided it into pixel correlation and uncorrelation:

$$
f_{j}(X)=w_{j}(X) f(X)
$$

$w_{j}(\mathrm{X})$ is a flux response function, and sampling path space by some distribution, that is to solve the integral using Monte Carlo method. Supposing $p=(1 / b) f$, where $b=\int_{\Omega} f(X) d \mu(X)$ is the integral of scalar contribution function, then to compute radiant flux of each pixel as following:

$$
\Phi\left(S_{j}\right) \approx \frac{1}{N} \sum_{i=1}^{N} b w_{j}\left(X_{i}\right)
$$

Only when $i \rightarrow \infty$, it will tends to equilibrium distribution.

\section{Improvement in Metropolis light transport}

Because start-up bias affect the convergence speed of Metropolis method, and the extension for path space is local by mutating current path to make small change. Besides, the optimal parameter setting often scene depended, and inappropriate settings will reduce convergence. Metropolis sampling is based on one probability distribution determined by the importance function to compute acceptance probability.

\section{Start-up Bias}

Analysis from Theory and Simulation Experiment

Sizmay et al. analyzed the start-up bias question from theory and simulation experiment [7]. First, an easy one-dimensional model was established and supposed the importance function as constant. Then the convergence of Metropolis sampling is examined by Fourier analysis. Finally, they confirmed that start-up bias will make bright or dark anomalies in final image using two different integral forms (BDPT and Ray-bundle transport).

Determined randomly sampling seeds

First, to get initial sample seed $\mathrm{X}_{0}$ according to the known distribution $p_{0}$ (e.g. BDPT) to sample path space. Because $p_{0}$ is not proportional to $f$, so should give a weight $W_{0}$ to start-up seed $\mathrm{X}_{0}$ :

$$
W_{0}=f\left(X_{0}\right) / p_{0}\left(X_{0}\right)
$$

In fact, only one path will be generated, and is likely to be degraded, then the weight of the subsequent sample is 0 . It is useful to do many times sampling by BDPT for solving the over big variance which can obtain $n$ paths $\mathrm{X}_{1}{ }^{(1)}, \ldots, \mathrm{X}_{\mathrm{N}}{ }^{(\mathrm{n})}$, and the weight of each path is:

$$
W_{0}^{(i)}=f\left(X_{0}^{(i)}\right) / p_{0}\left(X_{0}^{(i)}\right)
$$

Connecting the eye and light resource subpath to obtain $n$ paths. Second, defining the average weight of $m$ path as the weight of $n$ start-up seeds. Then to mutate from these seeds.

\section{Mutation Strategy}

Existing mutation strategies: bidirectional mutation, perturbation (e.g. lens perturbation, caustic perturbation and multiple chain perturbation), scattering and propagation mutation [8] and so on. Improved mutation strategies are as following.

Energy Redistribution Path Tracing (ERPT)

ERPT as proposed by Cline [9] is an extension of MLT. The core of ERPT is to choose an energy flow filter to redistribute the energy of initial Monte Carlo sampling points. First, using PT to choose multiple seed paths in each pixel. Then applying defined mutation strategy to the start-up seeds to generate new paths with accepted or not depending on acceptance probability, and give them part of energy in initial seeds.

Primary Sample Space MLT (PSSMLT)

Each sample can be described as a list of random numbers and a mapping from random numbers to the actual path. Kelemen et al. proposed an alternative mutation strategy called PSSMLT [10]. The algorithm only need one sampler to convert the list of random numbers to path, and calculate the 
whole contribution. PSSMLT not only reduce the variance, but also increase the acceptance probability, which can speed convergence. Besides, No complex techniques and proper parameters settings for PSSMLT.

Gradient-Domain MLT (GDMLT)

Lehtimen et al. introduce a novel Metropolis rendering algorithm GDMLT [11] that directly computes image gradients, and reconstructs the final image from the gradients by solving a Poisson equation. To guarantee both the gradients and the coarse image are adequately sampled [12], they controlled Metropolis sampling according to the contribution. Firstly, defining a generalized throughput function. Then defining the target function $f(z)$ as a combination of both generalized throughput $f^{*}(z)$ and base path throughput $f^{*}(x)$, and $f^{*}(z)$ can be negative [13]. So the target are defined as following:

$$
f(z)=\left\|f^{*}(z)\right\|+\alpha\left(\frac{1}{4}\left\|f^{*}(x)\right\|\right)
$$

Where $\alpha$ control the relative importance of the two terms. Once having the gradient images and a rough estimate of the actual image, then use a screened Poisson solver to find the image $I$ whose gradients best match the inputs [14,15]. GDMLT benefits from Manifold Exploration (ME) [16] which can improve the handling of specular and highly glossy paths and focus high density sampling on a smll part of path space.

Multiplexed Metropolis Light Transport (MMLT)

Samples generated as a history of Markov chain states are distributed according to the contribution to the image. Hechisuka et al. developed a novel rendering algorithm, Multiplexed Metropolis Light Transport (MMLT) [17], which adaptively construct paths with appropriate techniques as predicted by Multiple Importance Sampling (MIS) [18]. The algorithm not only explore the sample space using a Markov chain, but also the different estimators to generate samples.

\section{Parameter Control}

High performance rendering algorithms often have many control parameters that need to be set before rendering [19]. Therefore, the optimal values are scene dependent. Zsolnai et al. proposed a method to automatically control the large step probability parameter of Primary Sample Space Metropolis Light Transport (PSSMLT) [20], which does not need the extra computation time or pre-processing, and runs in parallel with the initial phase of the rendering. During the process, it gathers statistics from the process and computes parameters for the remaining part of the sample generation. The results show that the proposed theory value is very similar to the optimal value manually found in several complex scenes.

\section{Importance Function}

The distribution of sample in MLT is determined by importance function. In generally, describing $f(X)$ as the importance of sample X. Veach et al. proposed Two-Stage MLT [6]. Then Hoberock et al. proposed Multi-Stage MLT by iteratively guiding extension [21]. Therefore, Hoberock et al. also extended different importance functions and proposed Noise Aware MLT [21] which repeatedly adapting the importance function based on a progressive estimate of the noise distribution. The method lead to a better stratification of samples over the image plane, reducing visible noise. But new start-up bias is introduced after repeatedly updating the importance function.

\section{Conclusion}

The paper summarized the typical improvements for four main factors. MLT can better extend paths with high contribution to final image for indirect illumination or caustic scene. ME can make up insufficient of mutation strategies effectively, and improve the efficiency for glossy path transport. MMLT can effectively capture glossy paths. For difficult specular transport, GDMLT preforms better 
than other. In addition, ERPT can strive to stratify perturbations to avoid spot. Noise Aware MLT use the arbitrarily importance function to lock the visual noise in dark scene to generate more ideal image. Thus, how to select appropriate mutation to sample path in different scenes and change the form of acceptance probability to gain better result are still important question currently.

\section{References}

[1] Kajiya J T. The rendering equation[C]//ACM Siggraph Computer Graphics. ACM, 1986, 20(4): 143-150.

[2] Veach E, Guibas L J. Metropolis light transport[C]//Proceedings of the 24th annual conference on Computer graphics and interactive techniques. ACM Press/Addison-Wesley Publishing Co., 1997: 65-76.

[3] BAO Shi-qiang. The study of Bidirectional Paht Tracing based Metropolis Global Illumination Method[D]. Tianjin University, 2007.

[4] Metropolis N, Rosenbluth A W, Rosenbluth M N, et al. Equation of state calculations by fast computing machines[J]. The journal of chemical physics, 1953, 21(6): 1087-1092.

[5] Jensen H W, Arvo J, Fajardo M, et al. State of the art in Monte Carlo ray tracing for realistic image synthesis[J]. SIGGRAPH 2001 Course Notes, 2001.

[6] VEACH E.: Robust Monte Carlo Methods for Light transport Simulation [D]. PhD thesis, Stanford University, 1998.

[7] Szirmay-Kalos L, Dornbach P, Purgathofer W. On the start-up bias problem of metropolis sampling[J]. Winter School of Computer Graphics' 99, 1999: 273-280.

[8] Pauly, M, Kollig, T, Keller. A. Metropolis light transport for participating media [J]. Proceedings of the Euro graphics Workshop on Rendering Techniques. 2000, 11-22.

[9] Cline D, Talbot J, Egbert P. Energy redistribution path tracing[J]. ACM Transactions on Graphics (TOG), 2005, 24(3): 1186-1195.

[10]Kelemen C, Szirmay-Kalos L, Antal G, et al. A simple and robust mutation strategy for the metropolis light transport algorithm[C]//Computer Graphics Forum. Blackwell Publishing, Inc, 2002, 21(3): 531-540.

[11]Lehtinen J, Karras T, Laine S, et al. Gradient-domain metropolis light transport[J]. ACM Transactions on Graphics (TOG), 2013, 32(4): 95.

[12]Bhat P, Zitnick C L, Cohen M, et al. Gradientshop: A gradient-domain optimization framework for image and video filtering[J]. ACM Transactions on Graphics (TOG), 2010, 29(2): 10.

[13]Levin A, Zomet A, Peleg S, et al. Seamless image stitching in the gradient domain[M]//Computer Vision-ECCV 2004. Springer Berlin Heidelberg, 2004: 377-389.

[14]Hašan $M$, Pellacini $F$, Bala $K$. Matrix row-column sampling for the many-light problem[C]//ACM Transactions on Graphics (TOG). ACM, 2007, 26(3): 26.

[15] Ou J, Pellacini F. LightSlice: matrix slice sampling for the many-lights problem[J]. ACM Trans. Graph., 2011, 30(6): 179.

[16]Jakob W, Marschner S. Manifold Exploration: a Markov Chain Monte Carlo technique for rendering scenes with difficult specular transport[J]. ACM Transactions on Graphics (TOG), 2012, 31(4): 58.

[17]Georgiev I, Křivánek J, Slusallek P. Bidirectional light transport with vertex merging[C]//SIGGRAPH Asia 2011 Sketches. ACM, 2011: 27.

[18]Hachisuka T, Kaplanyan A S, Dachsbacher C. Multiplexed Metropolis Light Transport [J]. ACM Transactions on Graphics. 2014, 33(4):100:1-100:10.

[19]RITSCHEL T., DACHSBACHER C., GROSCH T., KAUTZ J. The State of the Art in Interactive Global Illumination [J]. Computer Graphics Forum. 2012, 31(1):160-188.

[20]Zsolnai K, Szirmay-Kalos L. Automatic Parameter Control for Metropolis Light Transport[C]//Eurographics 2013-Short Papers. The Eurographics Association, 2013: 53-56.

[21] Hoberok j, Hart J C. Arbitrary importance function for metropolis light transport[c]//Computer 
Graphics Forum. Blackwell Publishing Ltd, 2010, 29(6): 1993-2003. 